The ERS designates this

educational activity for a

maximum of 1 CME credit. For

information on how to earn

CME credits, see page 376 , or

visit www.ers-education.org/

breathe-cme.htm

\title{
Integrated care of the COPD patient: a pulmonary rehabilitation perspective
}

\author{
R. ZuWallack ${ }^{1}$ \\ L. Nici ${ }^{2}$ \\ ${ }^{1}$ St Francis Hospital, Hartford, CT, \\ and ${ }^{2}$ Providence VA Medical Center, \\ Providence, RI, USA.
}

\section{Educational aims}

1 To demonstrate that the current care system for chronic obstructive pulmonary disease (COPD) patients often falls short of desired outcomes.

1 To describe the concepts of the chronic care model and integrated care.

I To emphasise the positioning of pulmonary rehabilitation and self-management and self management strategies within integrated care.

\section{Summary}

The current acute care model for COPD is, in general, insufficient for optimal management of the disease. Single disease guidelines often fall short for diseases such as COPD that have prominent systemic manifestations and frequent comorbidities contributing to disease burden. Coordination of services is often inadequate, especially at the time of the COPD exacerbation, which is characterised by high morbidity, increased healthcare utilisation and increased mortality risk. The integrated care model for COPD may reduce some of these inefficiencies. On one level, integrated care means giving the right patient the right therapy at the right time. This can include smoking cessation therapies, the promotion of a healthy lifestyle, including regular exercise and increased activity, optimal pharmacotherapy, collaborative self-management strategies, palliative therapy and end-of-life care. Additionally, integrated care improves access to care and reduces fragmentation of care through partnering, communication and coordination among healthcare professionals, patients and their families. Integration at the time of the exacerbation includes a comprehensive assessment of the patient at the time of hospital discharge, proper discharge planning, early discharge senvices, a self-management plan, sharing this plan with all pertinent healthcare providers, utilisation of a professional for case management and facilitation of the exchange of information with modern information technology. Pulmonary rehabilitation provides many of these services and can be considered a component of integrated care.

\section{Introduction: a case presentation}

A 75-yrold ex-smoker with symptomatic COPD for $\geq 10$ yrs (patient $A$ ) was hospitalised for a COPD exacerbation. Patient A had been hospitalised 6 weeks earlier for an exacerbation, but did not fully recover. Other comorbidities included noninsulin-dependent diabetes mellitus, systemic hypertension, obesity, sleep apnoea and coronary artery disease (he had undergone a successful stent procedure 2 yrs earlier). Spirometry performed 3 yrs earlier was consistent with severe COPD: postbronchodilator forced expiratory volume in

\section{Correspondence}

R. ZuWallack

Section of Pulmonary and

Critical Care

St Francis Hospital \& Medical

Center

114 Woodland St

Hartford, CT 06105

USA

rzuwalla@stfranciscare.org

Provenance

Commissioned article,

peer reviewed.

Competing interests

$R$. ZuWallack has received speaking honoraria from Boehringer Ingelheim, Pfizer, AstraZeneca and GlaxoSmithKline. 
$1 \mathrm{~s}(\mathrm{FEV} 1) /$ forced vital capacity ratio 0.52 and FEV $138 \%$ predicted. His COPD was characterised by frequent exacerbations, resulting in three hospitalisations in the previous year. He visits his cardiologist, primary care physician and respirologist approximately once, three and four times per year, respectively.

In hospital, patient A was successfully treated for COPD and new-onset congestive heart failure (CHF) by his hospital physician (whom he has never seen before) and by his cardiologist's associate. Patient $A$ was discharged after 5 days in hospital with a printed list of medications and discharge directives. New medications included a course of oral corticosteroids with a tapering dose schedule and five additional days on antibiotics. Prior to discharge, the staff demonstrated that he could walk down the corridor on his own without supplemental oxygen. However, he was considerably weaker and more dyspnoeic than normal at baseline. A follow-up appointment with his primary care physician was organised in 3 weeks.

\section{The problem}

We believe this case presentation is typical of healthcare delivery for an individual with advanced COPD in the USA, which is probably not unlike medical care in other parts of the developed world. This system is not ideal. The following discussion focuses on some important issues that are often unaddressed and contribute to sub-

\section{Figure 1}

Systemic manifestations and common comorbidities in COPD [5-7].

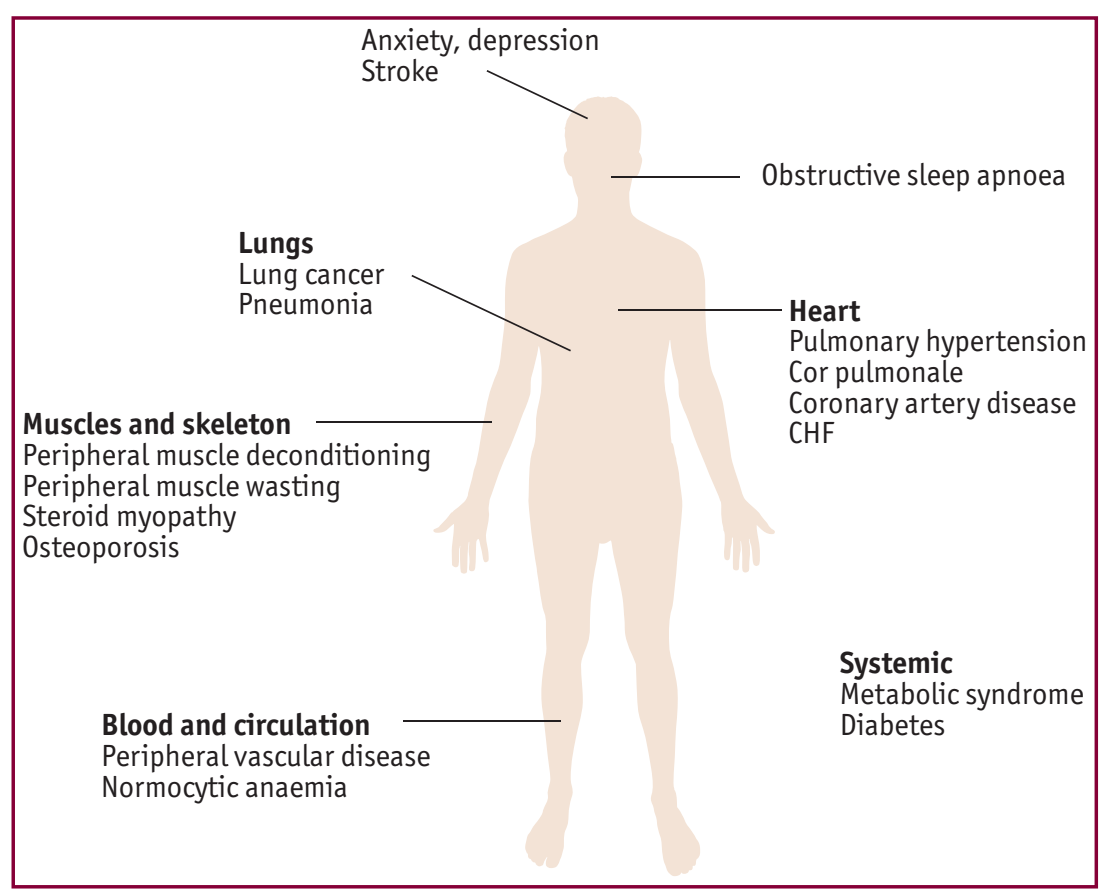

\section{The impact of COPD on quality of life and healthcare utilisation}

COPD is defined by the presence of airflow limitation that is not fully reversible $[1,2]$. Both the Global Initiative for Obstructive Lung Disease and the American Thoracic Society (ATS)/European Respiratory Society (ERS) guidelines state that COPD may have extrapulmonary effects (conditions) that can contribute to its morbidity $[1,2]$. Extrapulmonary conditions can be divided into systemic manifestations, such as peripheral muscle dysfunction and common comorbidities, e.g. lung cancer. However, this distinction is arbitrary. For example, smoking tobacco is a common and potent aetiologic agent for many of these conditions; spill-over systemic inflammation from COPD itself may contribute to systemic effects and comorbidities $[3,4]$. For the purpose of this review, we are combining systemic effects and common comorbidities under the heading of extrapulmonary conditions. Figure 1 lists extrapulmonary conditions frequently present in patients with COPD.

Patient A suffered from diabetes, hypertension, obesity, sleep apnoea, coronary artery disease, a recent history of CHF and possible steroid myopathy. These extrapulmonary conditions are prominent in morbidity and mortality in COPD [5] and may pose a greater mortality risk than COPD itself, especially in patients with less advanced disease. Therefore, it is naïve and potentially detrimental to the patient to focus treatment solely on the respiratory disease and to ignore the extrapulmonary conditions; however, this is a common occurrence.

\section{Single disease guidelines}

Basing clinical management on single disease guidelines often falls short and may result in undesirable effects in the patient. The COPD patient has, on average, 3.7 other chronic medical conditions compared with 1.8 for patients with other chronic illnesses [8]. The effects of these multiple chronic conditions and their treatments negatively interact [9], and the total morbidity is greater than the sum of the individual conditions.

In a recent analysis by Bord et al. [10], applying individual clinical practice guidelines to a hypothetical 79-yrold female with COPD, diabetes mellitus, osteoporosis, hypertension and 
osteoarthritis would result in prescribing 12 medications costing $\$ 406$ per month. Polypharmacy such as this, besides being expensive, promotes adverse drug reactions and other problems. This complexity calls for a more holistic and less fragmented approach.

\section{The current acute care model of healthcare}

Treatment for patient $\mathrm{A}$ focused on his acute exacerbation of COPD and CHF. Other important issues are likely to have been ignored. Furthermore, there will probably be relatively little communication among specialists and primary care providers, virtually no interdisciplinary decision making and little promotion of collaborative selfmanagement strategies. Based on this reality, it is clear our current acute care model for chronic diseases is insufficient for optimal management [11, 12]. Other models of care have been proposed, which focus on the individual, are proactive and evidencebased, foster collaborative selfmanagement and integrate services across providers (table 1) [13]. Many of these models are almost identical with a primary goal of improving outcomes in the frail elderly and other groups with complex needs.

\section{The chronic care model}

BODENHEIMER et al. [14] described the situation where acute symptoms and concerns are of higher importance than optimal chronic disease management as the "tyranny of the urgent". These authors proposed a chronic care model for the primary care of patients with chronic illnesses, such as COPD. This model has six components, which are listed in table 2. A chronic care model for COPD must integrate senvices and therapies across settings and providers and tailor therapy

\section{Table 1 Models of care terminology [13]}

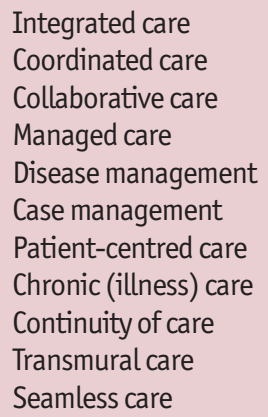

\section{Table 2 Chronic care model}

\section{Self-management support \\ Clinical information systems \\ Delivery system redesign \\ Decision support \\ Healthcare organisation \\ Community resourses}

to the individual needs of the patient. Which of the six components of this chronic care model listed in table 3 are necessary for optimal care in COPD patients has not yet been determined, although it appears that incorporating two or more components is needed to show beneficial effects in the area of healthcare utilisation [15].

\section{Integrated care, self-management and pulmonary rehabilitation}

\section{Integrated care}

The optimal treatment for COPD patients must concurrently address symptoms and disability from the respiratory disease and from its extrapulmonary conditions, adhering to principles of the chronic care model. Coordination of services is especially important at the time of the COPD exacerbation, which is characterised by high morbidity, high healthcare utilisation and even worse

Table 3 What a typical comprehensive, patient-centred, pulmonary rehabilitation programme provides

Comprehensive assessment by a multidisciplinary team with feedback to specialists and primary care providers

Exercise training leading to decreased dyspnoea and increased exercise capacity

Self-management education, including smoking cessation intervention, when indicated promotion of adherence with prescribed therapy and a collaborative action plan for the COPD exacerbation

Optimisation of pharmacotherapy and supplemental oxygen therapy

The promotion of a healthy lifestyle, including increased physical activity in the home setting, proper nutrition, recommended vaccinations

Symptom management techniques such as proper pacing and pursed-lip breathing

Psychosocial screening and support

End-of-life education and advance directives

Long-term exercise maintenance programmes with professional support

Outcome analysis, often including measurements of dyspnoea, health status and exercise capacity 
fragmentation of care. The integrated care model addresses these issues. Selfmanagement strategies and pulmonary rehabilitation are two important components of integrated care for COPD.

The World Health Organization (WHO) defines integrated care as " $a$ concept bringing together inputs, delivery, management and organisation of senvices related to diagnosis, treatment, care, rehabilitation and health promotion" [16]. An alternative definition by Mur-Veeman et al. [17] is "an organisational process of coordination that seeks to achieve seamless and continuous care, tailored to the patient's needs and based on a holistic view of the patient" [18]. These two definitions are complementary in that the WHO definition is system centred, stressing the coordination of services, while the Mur-VeEman et al. [17] definition focuses on the patient and the tailoring of care to individual patient needs. Wouters and VANDERHOVEN [19] consider integrated care in the context of a complex adaptive system, which is " a collection of individual agents with freedom to act in ways that are not always totally predictable, and whose actions are interconnected so that the action of one part changes the context of the other" [20]. Perhaps the simplest view is that integrated care consists of: 1) comprehensive intervention; 2) a self-management education programme; 3) an individually tailored care plan; and 4) increased accessibility to healthcare professionals [21].

A prominent goal of integrated care is improved access and decreased fragmentation of care, thereby increasing efficiency and quality of care and resulting in better outcomes and greater user satisfaction. This requires partnering, communication and coordination among healthcare professionals, patients and their families. The integration of services can be horizontal (e.g. among multi-hospital systems at the same level of healthcare delivery) or vertical (at different hierarchical levels such as hospital-based specialists, primary care providers, outpatient pulmonary rehabilitation staff and homecare professionals) [13]. There is ongoing discussion about whether the needs of a patient with a specific disease would be better served by a specialist, who has more knowledge and experience of that particular disease, or a primary care clinician, who is aware of the patient's history [22]. Integrated care transcends this argument by stating that both are necessary for optimal care and our goal should be to organise these services and establish better lines of communication.

Citing a WHO report [23], WouteRs and VANDERHOVEN [19] stress five core competencies that may complement existing care: 1) the ability to organise workforce care around the patient; 2) increase provider communication skills; 3) skills to ensure continuous improvement of quality and safety of care; 4) skills to monitor patients longitudinally; and 5) the ability to view care from the broadest perspective, including its multiple levels along the care continuum.

While there is considerable overlap between the concepts of integrated care and disease management, they do differ somewhat. Unlike the integrated care model, disease management traditionally targets patients with a single disease or condition, emphasising medication adherence and behaviour change to improve outcomes, especially healthcare utilisation. Concepts of disease management are now much broader in scope and more closely resemble integrated care [13].

\section{For the COPD patient}

For COPD, an important aspect of integrated care is giving the patient the correct therapy at the right time. This can include smoking cessation, the promotion of a healthy lifestyle (including regular exercise and increased activity), optimal pharmacotherapy, collaborative self-management strategies, palliative therapy and end-oflife care. This care must be anticipatory, not reactive. It is especially pertinent at the time of the COPD exacerbation, when greater collaboration among healthcare professionals in the hospital and the community is required. Integration of care in this setting should include a comprehensive assessment of the patient at the time of hospital discharge, proper discharge planning, early discharge services, a collaborative selfmanagement plan at the time of discharge, sharing this plan with all invloved healthcare providers, utilisation of a professional for case management, and facilitation of the exchange of information with modern information technology.

\section{Self-management}

A prominent component of integrated care is self-management, which has three components: education, behavioural support and motivational support [15]. For COPD patients, self-management, in collaboration with healthcare providers, has a primary objective of promoting self efficacy through changing behaviour [24]. Higher levels of self efficacy will lead to positive behaviours, such as improved adherence with prescribed therapy (including regular exercise) and the adoption of a healthy lifestyle (including smoking cessation 
and increased physical activity). Collaborative self-management is especially useful in early recognition and treatment of the COPD exacerbation, thereby reducing subsequent morbidity and healthcare utilisation. The authors of a meta-analysis including 14 trials of self-management education in COPD concluded that this intervention is associated with a reduction in hospital admissions, although clear recommendations could not be made regarding the content or format of the programmes [25].

\section{Pulmonary rehabilitation}

The ATS/ERS statement on pulmonary rehabilitation defines pulmonary rehabilitation as "an evidence-based, multidisciplinary, and comprehensive intervention for patients with chronic respiratory diseases who are symptomatic and often have decreased daily life activities. Integrated into the individualised treatment of the patient, pulmonary rehabilitation is designed to reduce symptoms, optimise functional status, increase participation, and reduce healthcare costs through stabilising or reversing systemic manifestations of the disease" [26]. Pulmonary rehabilitation includes comprehensive patient assessment, exercise training, education, nutritional intervention and psychosocial support. The definition stresses the importance of pulmonary rehabilitation in integrated care of the respiratory patient. The ATS/ERS statement explains that pulmonary rehabilitation can be considered a spectrum of intervention strategies integrated into the lifelong management of the respiratory patient, and that these strategies involve a dynamic, active collaboration among the patient, family and healthcare provider [26].

Some of the interventions in pulmonary rehabilitation are listed in table 3 . When needed, these important interventions can (and should) be given progressively by primary care providers and specialists. The interventions are conveniently bundled in the comprehensive pulmonary rehabilitation programme. The structure of pulmonary rehabilitation and its holistic approach provide the opportunity to address the complex needs of the COPD patient and coordinate the necessary services and interventions. As such, the comprehensive, patient-centred therapy administered by the interdisciplinary pulmonary rehabilitation team is not unlike that of the chronic or integrated care models in its approach.

Pulmonary rehabilitation as a programme differs somewhat from the chronic and integrated care models. While it certainly highlights patient- centred holistic care and self-management support, traditionally it has not stressed the integration of services and therapies across settings and providers. Clinical information system interventions, healthcare organisation and development of community resources (such as registries) are generally not emphasised in pulmonary rehabilitation. Integrated care includes all aspects of pulmonary rehabilitation, but focuses on their integration. In essence, pulmonary rehabilitation is a prominent component of integrated care of the respiratory patient.

\section{Clinical trials evaluating integrated care}

In 2006, Casas et al. [21] reported on the effectiveness of integrated care on subsequent hospitalisations in patients discharged following an exacerbation of COPD. Integrated care consisted of a comprehensive evaluation of the patient at discharge, self-management education, development of an individually tailored plan and accessibility of a specialised nurse to patients and primary care providers, facilitated through a webbased process. Compared with standard care, this form of integrated care reduced subsequent hospital admissions by $\sim 50 \%$. A subsequent analysis determined that patients given this integrated care, scored better in self-management areas, including COPD knowledge, exacerbation identification, exacerbation early treatment, inhaler adherence and correct use of an inhaler [27].

Recently, Koff et al. [28] reported on the effectiveness of proactive integrated care for patients with severe or very severe COPD [28]. Their concept of proactive integrated care included four interventions: 1) disease-specific education; 2) teaching self-management strategies; 3) enhanced communication with study coordinators; and 4) remote home monitoring. Monitoring included measuring changes in symptoms, oxygen saturation, $\mathrm{FEV}_{1}$, and steps during the 6-min walk distance test. This was accomplished with a portable oximeter, an FEV1 monitor, an electronic pedometer and a small telecommunication device and technology platform (the Health Buddy system, Bosch Healthcare, MI, USA). 20 patients given proactive integrated care were compared with 20 patients given standard care. Proactive integrated care resulted in significantly improved health-related quality of life, earlier detection of COPD exacerbations and a trend toward a reduction in healthcare utilisation.

\section{Educational questions}

1. True or false? Singledisease clinical practice guidelines are well-suited for elderly patients with multiple medical problems.

2. The chronic care model includes:

a) Promotion of self-management.

b) Improved interdisciplinary communication through utilisation of clinical informational systems.

c) Delivery system redesign.

d) Utilisation of community resources.

e) All of the above.

3. True or false? Pulmonary rehabilitation can be considered a component of the integrated care of the COPD patient.

4. True or false? A study utilising an integrated care approach to the treatment of COPD patients discharged following an exacerbation demonstrated decreased subsequent health-care utilisation. 


\section{Conclusion}

The acute care model for treatment of patients with COPD and extrapulmonary conditions (such as patient A) clearly has sub-optimal outcomes. The integrated care approach is ideally suited to the management of chronic diseases, such as COPD. Integrated care: 1) is patient centred but not limited to the traditional boundaries of the disease; 2) is not overwhelmed by the complexities of the multi-morbid patient; 3) stresses important self-management strategies; 4) coordinates care; and 5) enhances lines of communication. Emerging data suggest integrated care improves clinical outcomes in COPD compared with standard care.

Pulmonary rehabilitation, with its interdisciplinary, comprehensive patient-centred approach is an excellent starting point for the implementation of integrated care in the COPD patient. At present it is probably the best treatment for patient $A$, who is symptomatic, functionally limited and at high risk for re-hospitalisation. Indeed, a recent meta-analysis of pulmonary rehabilitation following a COPD exacerbation (involving six trials and 219 patients) concluded that this intervention led to reduced subsequent hospitalisations, decreased mortality and improved quality of life [29].

Where do we go from here? The integrated care model can be further incorporated into pulmonary rehabilitation to optimise care. Currently, the typical COPD patient referred to pulmonary rehabilitation has relatively stable, moderate-tovery-severe disease. Expanding the referral base to include patients with less severe disease will allow more proactive care, and initiating pulmonary rehabilitation at the time of the exacerbation will bring in the services of an already present interdisciplinary team. Incorporating information technology systems into pulmonary rehabilitation could improve outcomes through enhancing vertical lines of communication and further reinforcing positive health behaviour change.

\section{References}

1. Global Strategy for Diagnosis. Global strategy for diagnosis, management, and prevention of COPD. www.goldcopd. com/Guidelineitem.asp?l1=2\&l2=1\&intId=2003 Date last accessed: March 19, 2010.

2. American Thoracic Society/European Respiratory Society. Standards for the diagnosis and management of patients with COPD. www.ers-education.org/media/2004/pdf/44029.pdf Date last accessed: March 10, 2010.

3. Fabbri LM, Luppi F, Beghe B, et al. Complex chronic comorbidities of COPD. Eur Respir J 2008; 31: 204-212.

4. Watz H, Waschki B, Kirsten A, et al. The metabolic syndrome in patients with chronic bronchitis and COPD. Chest 2009; 136: 1039-1046.

5. Barnes PJ, Celli BR. Systemic manifestations and comorbisites of COPD. Eur Respir J 2009; 33: 1165-1185.

6. Chatila WM, Thomashow BM, Minai OA, et al. Comorbidities in chronic obstructive pulmonary disease. Proc Am Thor Soc 2008; 5: 549-555.

7. Sin DD, Man JP, Man SF. The risk of osteoporosis in Caucasian men and females with obstructive airways disease. Am J Med 2003; 114: 10-14.

8. Sin DD, Anthonisen NR, Soriano JB, et al. Mortality in COPD: role of comorbidites. Eur Respir J 2006; 28: 1245-1257.

9. Upshur REG, Tracey S. Chronicity and complexity. Is what's good for the diseases always good for the patients? Can Fam Physician 2008; 54: 1655-1658.

10. Boyd CM, Darer J, Boult C, et al. Clinical practice guidelines and quality of care for older patients with multiple comorbid diseases implications for pay performance. JAMA 2005; 294: 716-724.

11. Holman $\mathrm{H}$, Lorig K. Patients as partners in managing chronic disease. Partnership is a prerequisite for effective and efficient health care. BM J 2000; 320: 526-257.

12. Simpson AC, Rocker GM. Advanced chronic obstructive pulmonary disease: rethinking models of care. QJM 2008; 101: 697-704.

13. Nolte E, McKee M. Integration and chronic care: a review. In: Notle E, McKee M, eds. Caring for people with chronic conditions. New York, European Observatory on Health Care Systems and Policies, 2008; pp. 64-89.

14. Bodenheinmer T, Wagner EH, Grumbach K. Improving primary care for patients with chronic illness. JAMA 2002; 288: $1775-1779$.

15. Adams SG, Smith PK, Allan PF, et al. Systematic review of the chronic care model in chronic obstructive pulmonary disease prevention and management. Arch Intern Med 2007; 167: 551-561.

16. World Health Organization. The world health report 1998. Life in the 21st century. A vision of all. Geneva. www. who.int/whr/1998/en/whr98_en.pdf Date created: 1998.

17. Mur-Veeman I, Hardy B, Steenbergen M, et al. Development of integrated care in England and the Netherlands: management across public boundaries. Health Policy 2003; 65: 227-241.

18. Ouwens $\mathrm{M}$, Wollersheim $\mathrm{H}$, Hermens $\mathrm{R}$, et al. Integrated care programmes for chronically ill patients: a review of systemic reviews. Intern $\mathrm{J}$ Qual Health Care 2005; 17: 141-146.

19. Wouters EFM, Vanderhoven IML. Pulmonary rehabilitation and integrated care. Semin Respir Crit Care Med 2009; 30: 713-720.

20. Plsek PE, Greenhalgh T. Complexity science: the challenge of complexity in health care. BMJ 2001; 323: 625-628.

21. Casas A, Troosters T, Garcia-Aymerich J, et al. Integrated care prevents hospitalisation for exacerbations in COPD patients. Eur Respir J 2006; 28: 123-130.

23. World Health Organization. Preparing a health care workforce for the 21st century. The century challenge of chronic condtions. www.who.int/chp/knowledge/publications/workforce_report.pdf Date created: 2005. Date last accessed: March 19, 2010. 
24. Bourbeau J, van der Palen J. Promoting effective self-management programmes to improve COPD. Eur Respir J 2009; 33: 461-463.

25. Efing T, Monninkhof EEM, van der Valk PP, et al. Self-management education for patients with chronic obstructive pulmonary disease. Cochrane Database Syst Rev 2007; 4: CD002990.

26. Nici L, Donner C, Wouters E, et al. American Thoracic Society /European Respiratory Society statement on pulmonary rehabilitation. Am J Respir Crit Care Med 2006; 173: 1390-1413.

27. Garcia-Aymerich J, Hernandez C, Alonso A, et al. Effects of an integrated care intervention on risk factors of COPD readmission. Respir Med 2007; 101: 1462-1469.

28. Koff PB, Jones RH, Cashman JM, et al. Proactive integrated care improves quality of life in patients with COPD. Eur Respir J 2009; 33: 1031-1038.

29. Puhan M, Scherplatz M, Troosters T, et al. Pulmonary rehabilitation following exacerbations of chronic obstructive pulmonary disease. Cochrane Database Syst Rev 2009; 1: CD005305. 